\title{
A Comparative Study on Open Circuit Voltage Models for Lithium-ion Batteries
}

\author{
Quan-Qing Yu' ${ }^{1}$, Rui Xiong ${ }^{1 *}\left(\mathbb{D}\right.$, Le -Yi Wang ${ }^{2}$ and Cheng Lin ${ }^{1}$
}

\begin{abstract}
The current research of state of charge (SoC) online estimation of lithium-ion battery (LiB) in electric vehicles (EVs) mainly focuses on adopting or improving of battery models and estimation filters. However, little attention has been paid to the accuracy of various open circuit voltage $(\mathrm{OCV})$ models for correcting the SoC with aid of the ampere-hour counting method. This paper presents a comprehensive comparison study on eighteen OCV models which cover the majority of models used in literature. The low-current OCV tests are conducted on the typical commercial $\mathrm{LiFePO}_{4} /$ graphite (LFP) and LiNiMnCoO 2 /graphite (NMC) cells to obtain the experimental OCV-SoC curves at different ambient temperature and aging stages. With selected OCV and SoC points from experimental OCV-SoC curves, the parameters of each OCV model are determined by curve fitting toolbox of MATLAB 2013. Then the fitting OCV-SoC curves based on diversified OCV models are also obtained. The indicator of root-mean-square error (RMSE) between the experimental data and fitted data is selected to evaluate the adaptabilities of these OCV models for their main features, advantages, and limitations. The sensitivities of OCV models to ambient temperatures, aging stages, numbers of data points, and SoC regions are studied for both NMC and LFP cells. Furthermore, the influences of these models on SoC estimation are discussed. Through a comprehensive comparison and analysis on OCV models, some recommendations in selecting OCV models for both NMC and LFP cells are given.
\end{abstract}

Keywords: State of charge, Open circuit voltage model, Lithium-ion battery, NMC, LFP

\section{Introduction}

Due to the global energy crisis and environmental deterioration, electric vehicles (EVs) have had an unprecedented development opportunity in recent years [1]. A large number of advanced batteries with higher power and energy densities are connected in series or parallels to provide required power and energy for EVs. Therefore, a smart battery management system (BMS) is critical for safe and reliable operations of EVs [2]. One of the basic BMS functions is to monitor and estimate the state of charge $(\mathrm{SoC})$ of lithium ion batteries in real time [3]. Among different approaches for estimating the SoC, the model-based methods have been used extensively for their self-correct ability $[4,5]$. The models require an

\footnotetext{
*Correspondence: rxiong@bit.edu.cn; rxiong@ieee.org

${ }^{1}$ National Engineering Laboratory for Electric Vehicles, School of Mechanical Engineering, Beijing Institute of Technology, Beijing 100081, China

Full list of author information is available at the end of the article
}

accurate open circuit voltage $(\mathrm{OCV})$ representation for correcting SoC calculation [6, 7].

The OCV equals the terminal voltage when there are no polarization effects or voltage drop on the internal impedance. The OCV is related to the battery SoC and this relationship is commonly obtained by offline OCV tests $[8,9]$. Due to the hysteresis effects of lithium ion batteries, the average values of the OCV charge curve and OCV discharge curve is defined as the experimental OCV curve [10]. The experimental OCV-SoC curves differ among battery types and vary with ambient temperatures, aging stages, and current rates. Therefore, the OCV model should represent experimental OCVs with high fidelity. So far, a large number of nonlinear functions have been proposed to represent the OCV-SoC relationship. Plett proposed an OCV model which contains a linear function [5], a power function, and a logarithmic function. Polynomial functions were added into the OCV models in Refs. [11-13]. Tong et al. [14] removed the power function from the OCV models. Subsequently, 
several references replaced the logarithmic functions from OCV models by exponential functions [15-17]. Zhang et al. [18] reported an OCV model that contains a linear function, an exponential function, and a logarithmic function. In addition, polynomial functions with different orders are developed to fit OCV data points obtained from offline OCV tests [19-21].

All the reported OCV models have been shown in their original publications to be suitable for SoC estimation under their selected operating conditions. However, there are few studies that provide a comprehensive comparison of these diversified OCV models. Hu et al. [21] compared five OCV models and the 6th order polynomial function was reported to be the most accurate OCV model among them. However, the study is only conducted on $\mathrm{LiFePO}_{4}$ (LFP) batteries. Its adaptability to other battery types needs to be further investigated. Moreover, sensitivities of these OCV models to ambient temperatures, aging stages, and numbers of data points remain largely an open issue. Only three OCV models were compared by Zhang et al. [18] from the viewpoints of sensitivities to ambient temperatures and aging stages, without exploring the sensitivities to numbers of data points. In addition, an overwhelming majority of these OCV models are proposed based on the experimental data of middle SoC regions between $10 \%$ and $90 \%$, and their applicability to the entire $\mathrm{SoC}$ region is worth further study.

The key contribution of this paper is using an innovative approach to give a systematic comparison on the practicality of diversified OCV models. First, adaptability of these OCV models to different battery types is investigated. Second, sensitivities to ambient temperatures, aging stages, numbers of data points, and SoC regions are studied. Third, the impacts of these models on SoC estimation are explored. Finally, suggestions about the selection of OCV models are given based on comparison analyses.

The remainder of this paper is organized as follows. In Section 2, the experimental setup, battery specifications, and experimental OCV-SoC curves under different ambient temperatures and aging stages for two types of lithium ion batteries are given. Section 3 introduces eighteen OCV models to be studied and compared. A systematic comparison of diversified OCV models is presented in Section 4, followed by the conclusions in Section 5 .

\section{Experiments}

\subsection{Experimental Setup}

The battery test bench in Refs. [11, 22] is used to carry out battery tests in this study. Considering the OCVSoC curves differ among battery types, typical commercial $\mathrm{LiFePO}_{4} /$ graphite (LFP) and $\mathrm{LiNiMnCoO} /$ graphite (NMC) lithium ion battery cells are selected as the test samples, and their basic specifications are given in Table 1. It's worth noting that both the nominal capacity and actual capacity are measured through capacity test under $25{ }^{\circ} \mathrm{C}$ [22]. The capacity losses of the aged NMC and LFP cells are $10.2 \%$ and $10.5 \%$, respectively.

\subsection{OCV Tests}

The low-current OCV tests are performed to obtain the experimental OCV-SoC curves [17]. The fully charged battery cells are discharged at a constant low rate of $0.05{ }^{\circ} \mathrm{C}$ until fully discharged. After rest $2 \mathrm{~h}$, the battery cells are then charged at the same current rate until the upper cutoff voltage is reached. Afterwards, the cells will be fully charged with constant current constant voltage (CCCV) method. The voltage at this low current rate reflects the OCV at a close-to-equilibrium status. Due to the $\mathrm{OCV}$ is independent of ambient temperatures and aging stages, the $\mathrm{OCV}$ tests are performed under three temperatures (i.e., $10{ }^{\circ} \mathrm{C}, 25^{\circ} \mathrm{C}$ and $40{ }^{\circ} \mathrm{C}$ ) and two aging stages (i.e., fresh and aged battery cells) in this study.

The average OCV-SoC curves between the measures obtained during charge and discharge are illustrated in Figure 1. The OCV-SoC curves of NMC cells shown in Figure 1(a) change dramatically as the SoCs drop to $0 \%$ and gradually increase between $10 \%$ and $100 \%$ SoC regions, but for LFP cells shown in Figure 1(b), the OCVSoC curves change dramatically as the SoCs drop to $0 \%$ and rise to $100 \%$, and there are wide flat OCV plateau in the middle SoC regions. Which indicate the OCV-SoC relationships differ among battery types and its adaptability to different battery types needs to be further investigated. The large OCV errors of NMC cells occur in the middle and low SoC regions, but for LFP cells, the large OCV errors occur in the whole SoC regions, especially in the low and high SoC regions. The large errors may be caused by the rest effects at difference operation conditions. Which confirmed that the OCV-SoC relationship is independent of ambient temperatures and aging stages. In addition, the OCV models may be sensitive to SoC regions and numbers of data points for the different features of experimental OCV-SoC curves.

Table 1 Basic specifications of the battery cells

\begin{tabular}{lllll}
\hline Cell & $\begin{array}{l}\text { Nominal } \\
\text { voltage } \\
\text { (V) }\end{array}$ & $\begin{array}{l}\text { Nominal } \\
\text { capacity } \\
\text { (Ah) }\end{array}$ & $\begin{array}{l}\text { Actual } \\
\text { capacity } \\
\text { (Ah) }\end{array}$ & Cutoff voltage (V) \\
\hline NMC (Fresh) & 3.7 & 25 & 28.40 & $2.5 / 4.2$ \\
NMC (Aged) & 3.7 & 25 & 25.51 & $2.5 / 4.2$ \\
LFP (Fresh) & 3.2 & 20 & 19.84 & $2.0 / 3.65$ \\
LFP (Aged) & 3.2 & 20 & 17.77 & $2.0 / 3.65$ \\
\hline
\end{tabular}



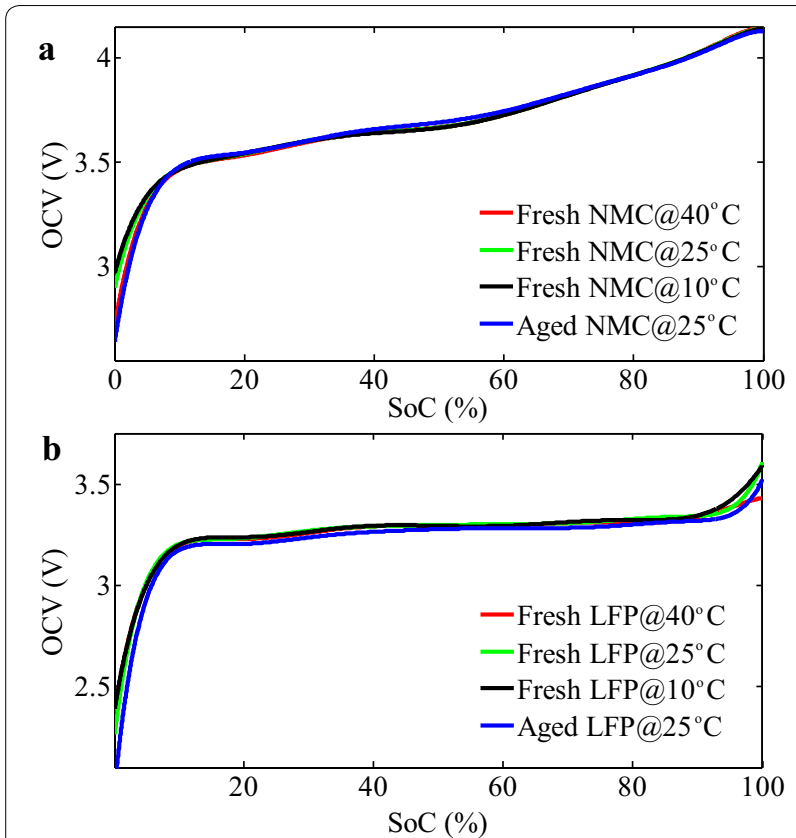

Figure 1 Experimental OCV-SoC curves at various temperatures and aging stages: a NMC cells, $\mathbf{b}$ LFP cells

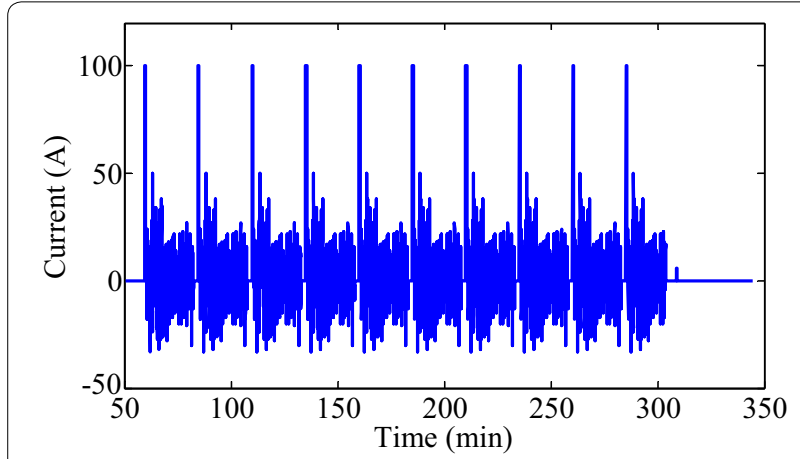

Figure 2 Current profile of the UDDS test

\subsection{UDDS Tests}

The urban dynamic driving sequence (UDDS) test is a typical driving test that is often used to evaluate the performance of the SoC estimation [23]. In this paper, the UDDS tests, as shown in Figure 2, are performed to evaluate the impact of OCV models on SoC estimation for both NMC and LFP fresh cells under $25^{\circ} \mathrm{C}$.

\section{OCV Model Structures}

A total of eighteen OCV models are selected from literature and compared in this study. Which cover the overwhelming majority of OCV models presented in the past studies. These OCV models are summarized in
Table 2. Noted that $U_{\mathrm{oc}}$ and $s$ are battery OCV and SoC, respectively. $K_{i}, \alpha_{i}, \beta_{i}, m$ and $n,(i=0,1,2, \ldots, 12)$ are the parameters of OCV models, which are determined by MATLAB curve fitting toolbox (Fit Option: Custom Equation; Robust: Bisquare; Algorithm: Trust-Region).

As shown in Table 2, these OCV models generally can be divided into four classes from the perspective of component terms.

1. A generalized polynomial function + a logarithmic function + a power function, such as model 1 to 5 ;

2. A generalized polynomial function + an exponential function, such as model 6 to 9;

3. A generalized polynomial function + an exponential function + a logarithmic function, such as model 10;

4. A generalized polynomial function, such as model 11 to 18 .

\section{Comparison and Analysis of OCV Models}

As shown in Table 2, there are distinct difference in structures of these OCV models. Some models are very simple with fewer numbers of parameters while some models are quite complicated with many parameters. Additionally, some models have limits on the SoC regions. In this section, we will give a comprehensive comparison and analysis on these OCV models, including the adaptabilities to battery types, sensitivities to temperatures, aging stages, SoC regions, numbers of data points, and impacts on SoC estimation. The fitted OCV should be as close to experimental OCV as possible. The fitting performance will be evaluated by the indicator of root-mean-square error (RMSE) between the experimental data and fitted data.

\subsection{Sensitivity to SoC Regions}

The most of OCV models focus on the middle SoC regions between $10 \%$ and $90 \%$, however, it is difficult to obtain accurate SoC initial value in real applications. Hence, if the parameters of OCV models are determined by fitting the experimental data of middle $\mathrm{SoC}$ regions (i.e., between $10 \%$ and $90 \%$ ), the OCVs will inaccurate in the low (i.e., $0 \%-10 \%$ ) and high $\mathrm{SoC}$ (i.e., 90\%-100\%) regions for their great features at these two regions, which will in turn contributed large SoC errors. Therefore, it is better to model the OCV by fitting the entire SoC region data.

Due to the logarithmic function exists in some OCV models, the entire or whole SoC region is selected from $0.001 \%$ to $99.999 \%$. The fitted OCV-SoC curves of different OCV models at $25{ }^{\circ} \mathrm{C}$ for both NMC and LFP fresh cells are shown in Figure 3. Note that the SoCs change dramatically when drop to $0 \%$ or rise to $100 \%$, the RMSEs are collected the SoC regions between $2.5 \%$ and $97.5 \%$. 
Table 2 OCV models evaluated in this study

\begin{tabular}{|c|c|c|}
\hline Model & Reference & OCV model expression \\
\hline 1 & [24-26] & $U_{o c}=K_{0}+K_{1} s+K_{2} / s+K_{3} \ln (s)+K_{4} \ln (1-s)$ \\
\hline 2 & {$[27,28]$} & $\begin{aligned} U_{o c} & =K_{0}+K_{1} s+K_{2} s^{2}+K_{3} / s+K_{4} \ln (s) \\
& +K_{5} \ln (1-s)\end{aligned}$ \\
\hline 3 & {$[11,12]$} & $\begin{aligned} U_{o c} & =K_{0}+K_{1} s+K_{2} s^{2}+K_{3} s^{3}+K_{4} / s+K_{5} \ln (s) \\
& +K_{6} \ln (1-s)\end{aligned}$ \\
\hline 4 & [14] & $U_{o c}=K_{0}+K_{1} s+K_{2} s^{2}+K_{3} s^{3}+K_{4} \ln (s)+K_{5} \ln (1-s)$ \\
\hline 5 & [29] & $U_{o c}=K_{0}+K_{1} \ln (s)+K_{2} \ln (1-s)$ \\
\hline 6 & [16] & $U_{o c}=K_{0}+K_{1} s+K_{2} s^{2}+K_{3} s^{3}+K_{4} \exp \left(K_{5} s\right)$ \\
\hline 7 & [15] & $U_{o c}=K_{0}+K_{1} s+K_{2}(1-\exp (\alpha s))+K_{3}\left(1-\exp \left(\beta(1-s)^{-1}\right)\right)$ \\
\hline 8 & [17] & $\begin{aligned} U_{\text {oc }} & =K_{0} s+K_{1}\left(1+\exp \left(\alpha_{1}\left(s-\beta_{1}\right)\right)^{-1}+K_{2}\left(1+\exp \left(\alpha_{2} s\right)\right)^{-1}\right. \\
& +K_{3}\left(1+\exp \left(\alpha_{3}\left(s-\beta_{2}\right)\right)^{-1}+K_{4}\left(1+\exp \left(\alpha_{4}(s-1)\right)^{-1}+K_{5}\right.\right.\end{aligned}$ \\
\hline 9 & {$[18,30]$} & $\left.U_{o c}=K_{0}+K_{1} s+K_{2}(1-\ln )\right)^{m}+K_{3} \exp (n(s-1))$ \\
\hline 10 & {$[14,31]$} & $U_{o c}=K_{0}+K_{1} s+K_{2} s^{2}$ \\
\hline 11 & {$[32]$} & $U_{\mathrm{OC}}=K_{0}+K_{1} s+K_{2} s^{2}+K_{3} s^{3}$ \\
\hline 12 & [20] & $U_{o c}=K_{0}+K_{1} s+K_{2} s^{2}+K_{3} s^{3}+K_{4} s^{4}$ \\
\hline 13 & [33] & $U_{o c}=K_{0}+K_{1} s+K_{2} s^{2}+K_{3} s^{3}+K_{4} s^{4}+K_{5} s^{5}$ \\
\hline 14 & {$[34,35]$} & $U_{o c}=K_{0}+K_{1} s+K_{2} s^{2}+K_{3} s^{3}+K_{4} s^{4}+K_{5} s^{5}+K_{6} s^{6}$ \\
\hline 15 & {$[36,37]$} & $U_{o c}=K_{0}+K_{1} s+K_{2} s^{2}+K_{3} s^{3}+K_{4} s^{4}+K_{5} s^{5}+K_{6} s^{6}+K_{7} s^{7}$ \\
\hline 16 & [38] & $\begin{aligned} U_{o c} & =K_{0}+K_{1} s+K_{2} s^{2}+K_{3} s^{3}+K_{4} s^{4}+K_{5} s^{5}+K_{6} s^{6}+K_{7} s^{7} \\
& +K_{8} s^{8}\end{aligned}$ \\
\hline 17 & {$[39,40]$} & $\begin{aligned} U_{o c} & =K_{0}+K_{1} s+K_{2} s^{2}+K_{3} s^{3}+K_{4} s^{4}+K_{5} s^{5}+K_{6} s^{6}+K_{7} s^{7} \\
& +K_{8} s^{8}+K_{9} s^{9}\end{aligned}$ \\
\hline 18 & [41] & $\begin{aligned} U_{o c} & =K_{0}+K_{1} s+K_{2} s^{2}+K_{3} s^{3}+K_{4} s^{4}+K_{5} s^{5}+K_{6} s^{6}+K_{7} s^{7} \\
& +K_{8} s^{8}+K_{9} s^{9}+K_{10} s^{10}+K_{11} s^{11}+K_{12} s^{12}\end{aligned}$ \\
\hline
\end{tabular}

The RMSEs of different SoC regions are given in Figure 4. Although these models perform well in fitting the SoC regions between $10 \%$ and $90 \%$, but some models perform poorly in fitting the entire SoC regions for both NMC and LFP fresh cells, especially in the low and high SoC regions.

Theoretically, the fitting performance is better with higher order polynomial function, however, the 2nd to 4th order polynomial functions perform well in middle SoC regions, but perform poorly in low and high SoC regions. But for 5 th and 6 th order polynomial functions, the OCV-SoC curves pass through data points $0 \%$ and $100 \%$ and fluctuate sharply in the middle SoC regions. The fitting performances are become better for the 7 th to 12 th order polynomial functions. Therefore, we may conclude from Figure 4 that the models 16, 17 and 18 are suitable for NMC cell and models 7, 17 and 18 are suitable for LFP cell, which perform well in both the entire and middle SoC regions. In the following sections, all the OCV models are determined from the experimental data of entire SoC regions.

\subsection{Sensitivity to Numbers of Data Points}

As mentioned above, the OCV models are determined by fitting certain numbers of experimental data points with MATLAB curve fitting toolbox. Some studies used 21 data points (i.e., in every $5 \%$ SoC interval) to determine the OCV model [13, 14, 42], but some other studies used 11 data points (i.e., in every $10 \%$ SoC interval) [43-45] or 51 data points (i.e., in every $2 \%$ SoC interval) [30] to determine the OCV models. The comparison results depicted in Figure 5 indicate that the OCV models are more sensitive to the data points for LFP cell than NMC cell. These may be caused by their differences in low and high SoC regions of OCV-SoC curves. In addition, the RMSEs of NMC cell change slightly with increasing the numbers of data points except the models 5, 7, 12 and 13, but for the LFP cell, the RMSEs are generally decrease greatly with 21 data points than with 11 data points, but the RMSEs virtually unchanged if change the number of data points from 21 to 51 except models 8 and 14 . Note that the models 8 and 18 

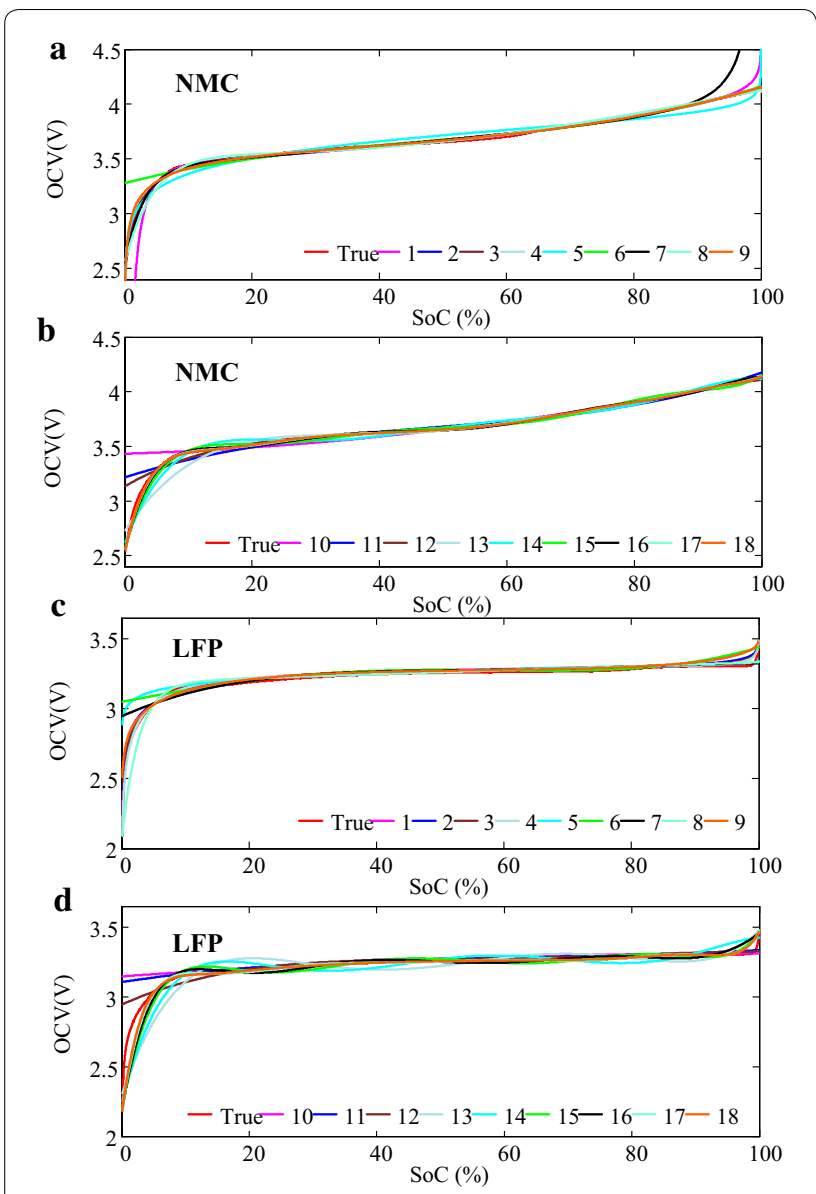

Figure 3 OCV-SoC curves of different models at $25^{\circ} \mathrm{C}$ : a fresh NMC cell (model 1-9), b fresh NMC cell (model 10-18), c fresh LFP cells (model 1-9), d fresh LFP cells (model 10-18)

require more than 12 and 13 data points, respectively, to fit by MATLAB curve fitting toolbox.

With the low-current OCV test, we can easily obtain certain numbers of data points, but for the other OCV test method, such as incremental OCV test, the experimental time will be greatly raised with increasing numbers of data points, therefore, it is recommended to model OCV by 21 data points by compromising the test time and model accuracy. In addition, we may conclude from Figure 5 that the models 4, 16 and 17 are suitable for NMC cell and models 4, 7 and 17 are suitable for LFP cell for their robustness against numbers of data points.

\subsection{Sensitivity to Ambient Temperatures}

The comparison results of OCV models at different ambient temperatures are illustrated in Figure 6. It is can be seen that most OCV models are sensitive to the ambient temperatures, especially the low temperature for both NMC and LFP cells. In addition, models 5 to 12 are more sensitive to temperatures than other models for NMC cell, but for the LFP cell, models 8,13 and 14 are more sensitive to temperatures than other models. Besides, a conclusion can be drawn from Figure 6 is that models 16, 17 and 18 are recommended for NMC cell and models 4, 17 and 18 are recommended for LFP cell for their robustness against ambient temperatures.

\subsection{Sensitivity to Aging Stages}

Not only the temperatures but also the battery aging stages can influence the battery OCV $[46,47]$. The comparison results of OCV models at different aging stages are illustrated in Figure 7. It is can be seen that most OCV models are sensitive to battery aging affects, especially models 5, 7, 8, 11 and 12 for NMC cells and models 8,13 and 14 for LFP cells. In addition, a conclusion can be drawn from Figure 7 is that models 3, 17 and 18 are recommended for NMC cells and models 4,17 and 18 are recommended for LFP cell for their robustness against aging affects.

\subsection{Impacts on SoC Estimation}

OCV model is commonly used to correct the SoC with aid of ampere-hour counting in SoC estimation process. In this study, the OCV can be regarded as part of the parameters of first RC battery model and can be identified by the $\mathrm{H}$ infinity filter $[22,38]$, then the OCV models only affect the SoC estimation process other than the parameters identification process. With the same online parameters delivered to SoC estimation process, it is more easily to observe the impacts of OCV models on SoC estimation with unscented Kalman filter (UKF) [22]. The results are shown in Figure 8. It is clear that these OCV models perform generally better for LFP than NMC cells except model 8 at $25^{\circ} \mathrm{C}$. In addition, a conclusion can be drawn from Figure 8 is that models 16, 17 and 18 are recommended for both NMC and LFP cells.

Based on the comparisons above, the compared fitting results of OCV models can be summarized in Table 3 . Model 18 is a little bit better than model 17 representing experimental OCV-SoC relationship. Considering quite a few studies will still choose 11 data points from incremental OCV test data to model the OCV-SoC relationship, which only suit to fit no more than 10th order polynomial functions. Hence, Model 17 is more suitable than model 18. Furthermore, one could speculate that the improvement of accuracy between 9 and 10 order polynomial functions can be quite small. Therefore, it can be concluded that model 17 , namely 9 th order polynomial function, is better than others for both NMC and LFP cells. And this study recommends 9th order polynomial function to model the OCV for both the NMC and LFP cells. 

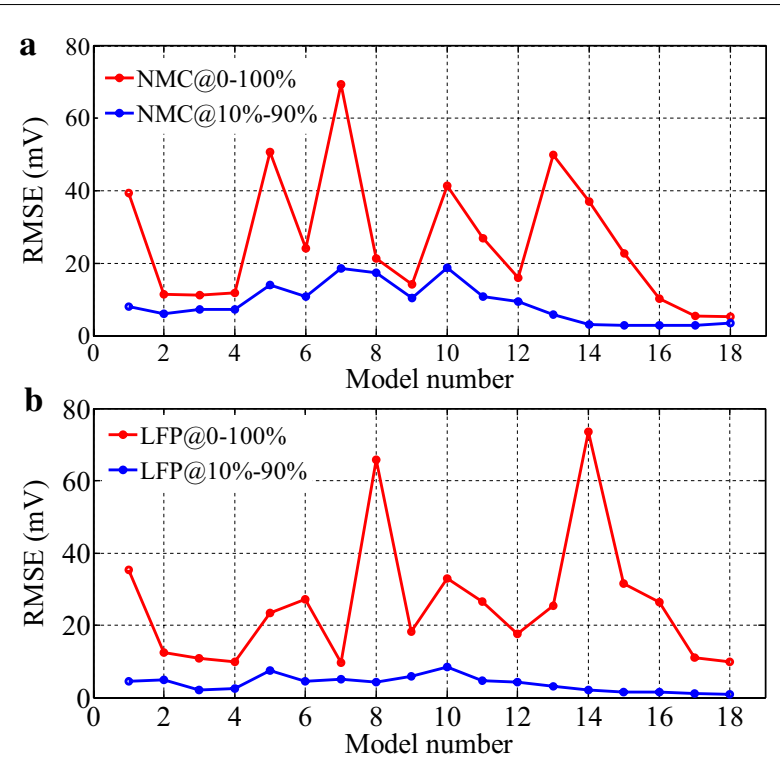

Figure 4 Comparison results of different $\mathrm{SoC}$ regions at $25^{\circ} \mathrm{C}$ : a fresh NMC cell, $\mathbf{b}$ fresh LFP cell
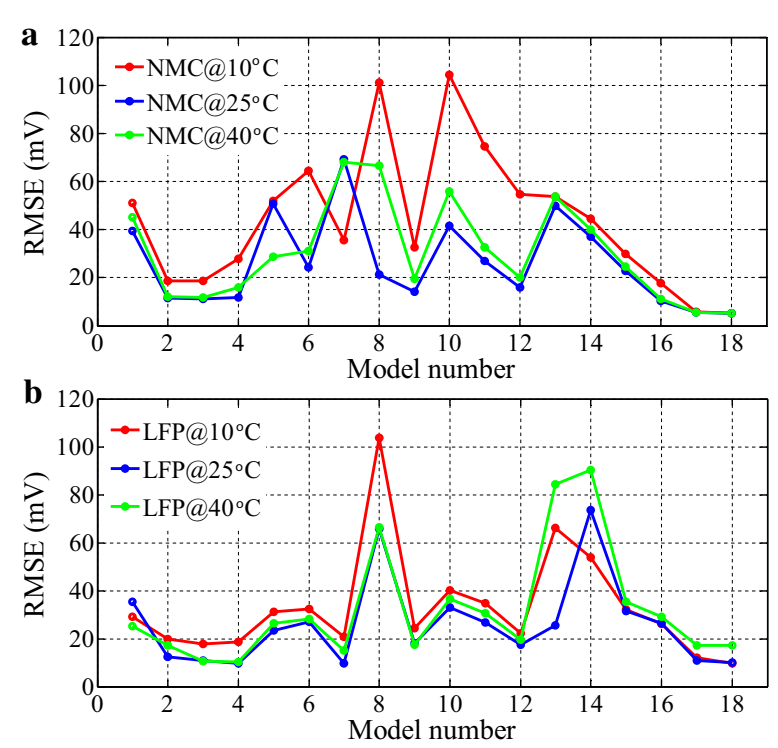

Figure 6 Comparison results of OCV modes at different temperatures: $\mathbf{a}$ fresh NMC cell, $\mathbf{b}$ fresh LFP cell

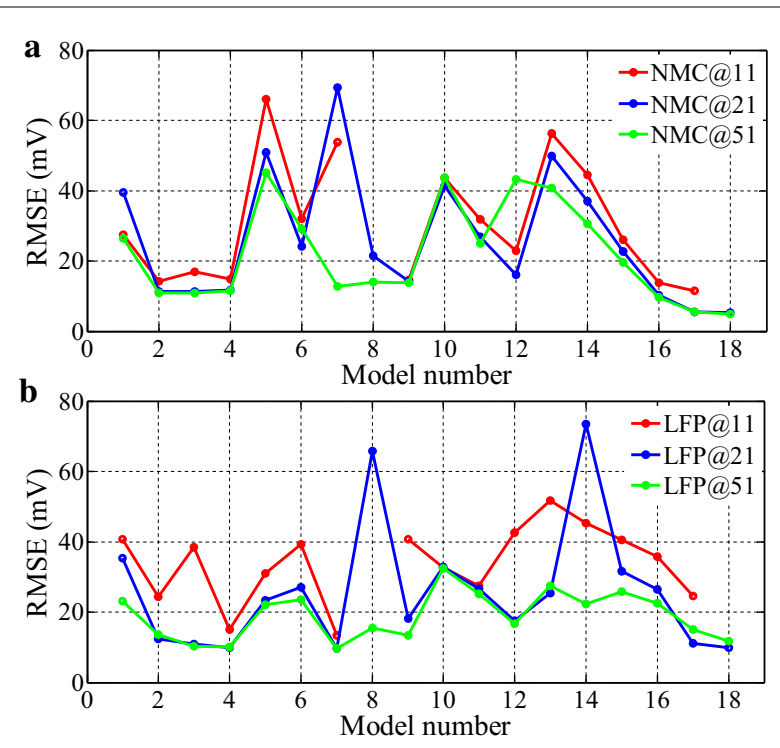

Figure $\mathbf{5}$ Comparison results of different numbers of data points at $25^{\circ} \mathrm{C}$ : a fresh NMC cell, $\mathbf{b}$ fresh LFP cell

\section{Conclusions}

1. This study conducts a systematic comparative study on eighteen OCV models. Low-current OCV tests are employed to obtain experimental OCV-SoC curves as the basis for comparison. The parameters of these OCV models are determined by experimental OCV data points.
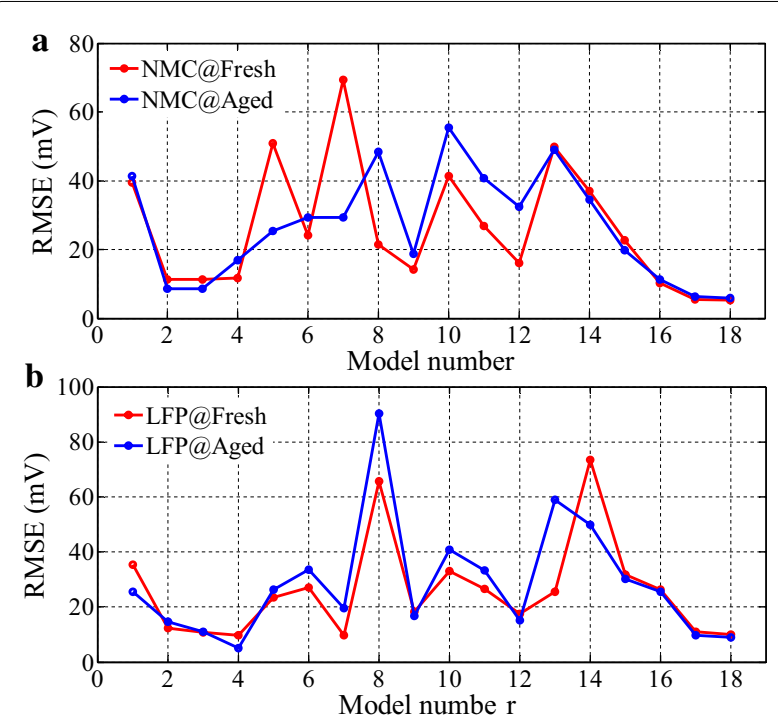

Figure $\mathbf{7}$ Comparison results of OCV modes at different aging stages: a NMC cells, b LFP cells

2. NMC and LFP cells are used to evaluate how well the OCV models can accurately represent the OCVSoC curves. Two representative experimental SoC regions $(0 \%-100 \%$ and $10 \%-90 \%)$ data are used to fit the OCV models. The results show that some OCV models perform poorer over the entire $\mathrm{SoC}$ region than the middle SoC region.

3. The data size plays an important role in model selection. A set of 21 data points is employed to compare 


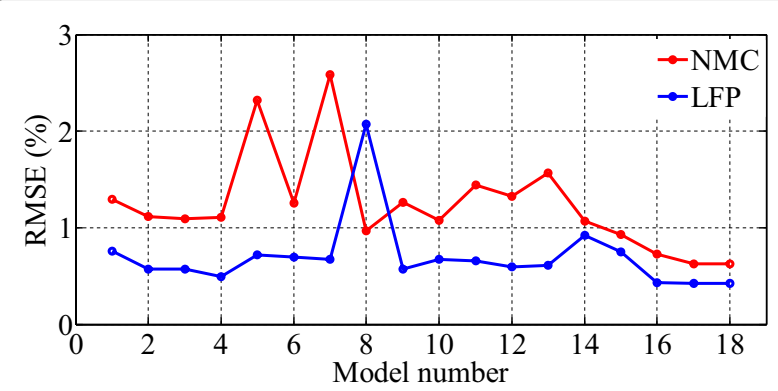

Figure 8 Impacts of OCV models on $\mathrm{SoC}$ estimation at $25^{\circ} \mathrm{C}$ for both NMC and LFP fresh cells

Table 3 Comprehensive comparison of OCV models

\begin{tabular}{|c|c|c|c|c|c|c|}
\hline Model number & 3 & 4 & 7 & 16 & 17 & 18 \\
\hline \multicolumn{7}{|l|}{ Sensitivity to SoC regions } \\
\hline \multicolumn{7}{|c|}{ Sensitivity to numbers of data points } \\
\hline \multicolumn{7}{|c|}{ Sensitivity to ambient temperatures } \\
\hline \multicolumn{7}{|l|}{ Sensitivity to aging stages } \\
\hline Impacts on SoC estimation & & & & & & \\
\hline
\end{tabular}

Note: Mark $\mathbf{\Delta}$ indicates the NMC cell and mark $\boldsymbol{\nabla}$ indicates the LFP cell

models with data size constraints. In addition, model sensitivity and robustness against temperatures and aging stages investigated. The results indicate that 9 th order polynomial model is more robust than other models. These conclusions are further validated by comparing model impact on SoC estimation.

4. By thorough analyses of $\mathrm{OCV}$ models, the 9th order polynomial model is recommended for both NMC and LFP cells.

\section{Authors' Contributions}

RX was in charge of the whole trial and computational work, proposed the OCV model and implemented the verification; Q-QY wrote the manuscript; L-YW corrected the language and gave some suggestions. CL assisted with sampling and laboratory analyses. All authors read and approved the final manuscript.

\section{Author details}

${ }^{1}$ National Engineering Laboratory for Electric Vehicles, School of Mechanical Engineering, Beijing Institute of Technology, Beijing 100081, China. ${ }^{2}$ Department of Electrical and Computer Engineering, Wayne State University, Detroit, MI 48202, USA.

\section{Authors' Information}

Quan-Qing Yu, born in 1984, is currently a PhD candidate at School of Mechanical Engineering, Beijing Institute of Technology, Beijing, China. He conducted scientific research as a joint Ph.D. student at Department of Electrical and Computer Engineering, Wayne State University, Detroit, Michigan, USA, between
2016 and 2017. He is currently a Research Scholar in the Center for Advanced Life Cycle Engineering (CALCE), University of Maryland, College Park. His research interests include battery state estimation, reliability, fault diagnosis and prognosis.

Rui Xiong, born in 1985, is currently an associate professor at Department of Vehicle Engineering, School of Mechanical Engineering, Beijing Institute of Technology, Beijing, China. He received his M.S. degree in vehicle engineering and Ph.D. degree in mechanical engineering from Beijing Institute of Technology, Beijing, China, in 2010 and 2014, respectively. He conducted scientific research as a joint Ph.D. student in the DOE GATE Center for Electric Drive Transportation at the University of Michigan, Dearborn, MI, USA, between 2012 and 2014. Since 2017, he has been an Adjunct Professor at Faculty of Science, Engineering and Technology, Swinburne University of Technology, Melbourne, Vic., Australia. He has conducted extensive research and authored more than 100 peer-reviewed articles. He holds eight patents. His research interests mainly include electrical/ hybrid vehicles, energy storage, and battery management system. He received the Excellent Doctoral Dissertation from Beijing Institute of Technology in 2014, the first prize of Chinese Automobile Industry Science and Technology Progress Award in October 2015 and the second prize of National Defense Technology Invention Award in December 2016. He received Best Paper Awards from the journal Energies and several International conferences. He is serving as the Associate Editors of IEEE Access and SAE International Journal of Alternative Powertrains, Editorial Board of the Applied Energy, Energies, Sustainability and Batteries. He was the conference chair of the 2017 International Symposium on Electric Vehicles held in Stockholm, Sweden.

Le-Yi Wang, born in 1955, is currently a professor at Department of Electrical and Computer Engineering, Wayne State University, Detroit, USA. He has been an IEEE Fellow since 2002. He received his PhD degree from Department of Electrical Engineering, McGill University, Montreal, Canada, in 1990. His research interests mainly include system identification, networked control systems, renewable energy technologies and electrical engineering.

Cheng Lin, born in 1968, is currently a professor and PhD Tutor at Deputy Director of National Engineering Laboratory for Electric Vehicle, Director of Research Center for Electric Vehicle in Beijing, Academic Leader of Collaborative Innovation Center of Electric Vehicles in Beijing, China. He received his B.S. and M.S. degree in mechanical engineering from Wuhan Institute of Technology, China, in 1990 and 1995, respectively. He received his Ph.D. degree in mechanical engineering from Beijing Institute of Technology, China, in 2002. From 2010 to 2011, he was a Senior Research Fellow sponsored by Country China Scholarship Council at Michigan University, Ann Arbor, USA. He has conducted extensive research in the field of electric vehicle. vehicle dynamics, and optimization and lightweight of automotive body structure.

\section{Competing Interests}

The authors declare no competing financial interests.

\section{Funding}

Supported by National Natural Science Foundation of China (Grant No. 51507012), and Beijing Municipal Natural Science Foundation of China (Grant No. 3182035). The systemic experiments of the lithium-ion batteries were performed at the Advanced Energy Storage and Application (AESA) Group, Beijing Institute of Technology.

\section{Publisher's Note}

Springer Nature remains neutral with regard to jurisdictional claims in published maps and institutional affiliations.

Received: 2 August 2017 Accepted: 7 Auqust 2018

Published online: 17 August 2018

\section{References}

[1] R Xiong, Y Zhang, $\mathrm{H} \mathrm{He}$, et al. A double-scale, particle-filtering, energy state prediction algorithm for lithium-ion batteries. IEEE Transactions on Industrial Electronics, 2018, 65(2):1526-1538.

[2] R Xiong, JY Cao, Q Q Yu, et al. Critical review on the battery state of charge estimation methods for electric vehicles. IEEE ACCESS, 2018, 6(1):1832-1843. 
[3] Y G Liu, J Li, DT Qin, et al. Parameter optimization of hybrid electric vehicle based on multi-cycle optimization algorithm. Journal of Mechanical Engineering, 2017, 53(16): 61-69. (in Chinese)

[4] Z B Wei, T M Lim, M Skyllas-Kazacos, et al. Online state of charge and model parameter co-estimation based on a novel multi-timescale estimator for vanadium redox flow battery. Applied Energy, 2016, 172: 169-179.

[5] G L Plett. Extended Kalman filtering for battery management systems of LiPB-based HEV battery packs-Part 2. Modeling and identification. Journal of Power Sources, 2004, 134(2): 262-276.

[6] W Waag, D U Sauer. Adaptive estimation of the electromotive force of the lithium-ion battery after current interruption for an accurate stateof-charge and capacity determination. Applied Energy, 2013, 111(4): 416-427.

[7] A Marongiu, F G W Nußbaum, W Waag, et al. Comprehensive study of the influence of aging on the hysteresis behavior of a lithium iron phosphate cathode-based lithium ion battery - An experimental investigation of the hysteresis. Applied Energy, 2016, 171: 629-645.

[8] Y Zheng, M G Ouyang, L Lu, et al. Understanding aging mechanisms in lithium-ion battery packs: From cell capacity loss to pack capacity evolution. Journal of Power Sources, 2015, 278: 287-295.

[9] C Lin, H Mu, R Xiong, et al. Multi-model probabilities based state fusion estimation method of lithium-ion battery for electric vehicles: State-ofenergy. Applied Energy, 2017, 194(15): 560-568.

[10] F Baronti, W Zamboni, R Roncella, et al. Open-circuit voltage measurement of Lithium-Iron-Phosphate batteries. IEEE International Instrumentation and Measurement Technology Conference, 2015: 1711-1716.

[11] R Xiong, F C Sun, HW He, et al. A data-driven adaptive state of charge and power capability joint estimator of lithium-ion polymer battery used in electric vehicles. Energy, 2013, 63(1): 295-308.

[12] R Xiong, F C Sun, Z Chen, et al. A data-driven multi-scale extended Kalman filtering based parameter and state estimation approach of lithium-ion polymer battery in electric vehicles. Applied Energy, 2014, 113(1): 463-476.

[13] G Z Dong, J W Wei, C B Zhang, et al. Online state of charge estimation and open circuit voltage hysteresis modeling of $\mathrm{LiFePO}_{4}$ battery using invariant imbedding method. Applied Energy, 2016, 162(1): 163-171.

[14] S J Tong, M P Klein, JW Park. On-line optimization of battery open circuit voltage for improved state-of-charge and state-of-health estimation. Journal of Power Sources, 2015, 293: 416-428.

[15] Y Hu, S Yurkovich, Y Guezennec, et al. Electro-thermal battery model identification for automotive applications. Journal of Power Sources, 2011, 196(1): 449-457.

[16] M Chen, S Member, G A Rinc. Accurate electrical battery model capable of predicting runtime and I-V performance. IEEE Transactions on Energy Conversion, 2006, 21(2): 504-511.

[17] C H Weng, J Sun, H Peng. A unified open-circuit-voltage model of lithiumion batteries for state-of-charge estimation and state-of-health monitoring. Journal of Power Sources, 2014, 258(14): 228-237.

[18] C P Zhang, J C Jiang, L Zhang, et al. A generalized SOC-OCV model for lithium-ion batteries and the SOC estimation for LNMCO battery. Energies, 2016, 9(11): 900

[19] K S Low, H Aung. Temperature dependent state-of-charge estimation of lithium ion battery using dual spherical unscented Kalman filter. IET Power Electronics, 2015, 8(10): 2026-2033.

[20] R Xiong, HW He, F C Sun, et al. Evaluation on state of charge estimation of batteries with adaptive extended Kalman filter by experiment approach. IEEE Transactions on Vehicular Technology, 2013, 62(1): 108-117.

[21] X S Hu, S Li, H Peng, et al. Robustness analysis of state-of-charge estimation methods for two types of Li-ion batteries. Journal of Power Sources, 2012, 217(11): 209-219.

[22] R Xiong, Q Q Yu, L Y Wang, et al. A novel method to obtain the open circuit voltage for the state of charge of lithium ion batteries in electric vehicles by using $\mathrm{H}$ infinity filter. Applied Energy, 2017, 207(1): 346-353.

[23] H Wu, S Yuan, X Zhang, et al. Model parameter estimation approach based on incremental analysis for lithium-ion batteries without using open circuit voltage. Journal of Power Sources, 2015, 287: 108-118.

[24] Y Wang, C Zhang, Z Chen. A method for state-of-charge estimation of LiFePO4 batteries at dynamic currents and temperatures using particle filter. Journal of Power Sources, 2015, 279: 306-311.

[25] X Liu, Z Chen, C Zhang, et al. A novel temperature-compensated model for power Li-ion batteries with dual-particle-filter state of charge estimation. Applied Energy, 2014, 123(2): 263-272.
[26] F Yang, Y Xing, D Wang, et al. A comparative study of three model-based algorithms for estimating state-of-charge of lithium-ion batteries under a new combined dynamic loading profile. Applied Energy, 2016, 164: 387-399.

[27] X Zhang, Y Wang, D Yang, et al. An on-line estimation of battery pack parameters and state-of-charge using dual filters based on pack model. Energy, 2016, 115: 219-229.

[28] G Dong, Z Chen, J Wei, et al. An online model-based method for state of energy estimation of lithium-ion batteries using dual filters. Journal of Power Sources, 2016, 301: 277-286.

[29] R Xiong, H W He, F C Sun, et al. Online estimation of peak power capability of Li-Ion batteries in electric vehicles by a hardware-in-loop approach. Energies, 2012, 5(5): 1455-1469.

[30] C P Zhang, LY Wang, X Li, et al. Robust and adaptive estimation of state of charge for lithium-ion batteries. IEEE Transactions on Industrial Electronics, 2015, 62(8): 4948-4957.

[31] HW He, X Rui, XW Zhang, et al. State-of-charge estimation of the lithium-ion battery using an adaptive extended Kalman filter based on an improved Thevenin model. IEEE Transactions on Vehicular Technology, 2011, 60(4): 1461-1469.

[32] Y Tian, D Li, J Tian, et al. State of charge estimation of lithium-ion batteries using an optimal adaptive gain nonlinear observer. Electrochimica Acta 2017, 225: 225-234

[33] KW E Cheng, B P Divakar, H Wu, et al. Battery-management system (BMS) and SOC development for electrical vehicles. IEEE Transactions on Vehicular Technology, 2011, 60(1): 76-88.

[34] A Szumanowski, Y H Chang. Battery management system based on battery nonlinear dynamics modeling. IEEE Transactions on Vehicular Technology, 2008, 57(3): 1425-1432.

[35] X S Hu, S Li, H Peng, et al. Charging time and loss optimization for LiNMC and LiFePO, batteries based on equivalent circuit models. Journal of Power Sources, 2013, 239(10): 449-457.

[36] H Aung, K S Low, et al. State-of-charge estimation of lithium-ion battery using square root spherical unscented Kalman filter (Sqrt-UKFST) in nanosatellite. IEEE Transactions Power Electronics, 2015, 30(9): 4774-4783.

[37] Y Tian, B Xia, W Sun, et al. A modified model based state of charge estimation of power lithium-ion batteries using unscented Kalman filter. Journal of Power Sources, 2014, 270(3): 619-626.

[38] Q Q Yu, R Xiong, C Lin, et al. Lithium-ion battery parameters and state-ofcharge joint estimation based on $\mathrm{H}$ infinity and unscented Kalman filters. IEEE Transactions on Vehicular Technology, 2017, 66(10): 8693-8701.

[39] A Sidhu, A Izadian, S Anwar. Adaptive nonlinear model-based fault diagnosis of Li-lon batteries. IEEE Transactions on Industrial Electronics, 2015, 62(2): 1002-1011.

[40] B Xia, C Chen, Y Tian, et al. A novel method for state of charge estimation of lithium-ion batteries using a nonlinear observer. Journal of Power Sources, 2014, 270(4): 359-366.

[41] S Zhao, S R Duncan, D A Howey. Observability analysis and state estimation of lithium-ion batteries in the presence of sensor biases. IEEE Transactions on Control Systems Technology, 2017, 25(1): 326-332.

[42] ZT Liu, HW He. Model-based sensor fault diagnosis of a lithium-ion battery in electric vehicles. Energies, 2015, 8(7): 6509-6527.

[43] S Nejad, DT Gladwin, D A Stone. A systematic review of lumped-parameter equivalent circuit models for real-time estimation of lithium-ion battery states. Journal of Power Sources, 2016, 316: 183-196

[44] Z Chen, Y Fu, C C Mi. State of charge estimation of lithium-ion batteries in electric drive vehicles using extended Kalman filtering. IEEE Transactions on Vehicular Technology, 2013, 62(3): 1020-1030.

[45] S Nejad, D T Gladwin, D A Stone. Enhanced state-of-charge estimation for lithium-ion iron phosphate cells with flat open-circuit voltage curves. Industrial Electronics Society, IECON 2015, Conference of the IEEE, 2016: 3187-3192

[46] Y Zou, X S Hu, H Ma, et al. Combined state of charge and state of health estimation over lithium-ion battery cell cycle lifespan for electric vehicles. Journal of Power Sources, 2015, 273: 793-803.

[47] A Farmann, D U Sauer. A study on the dependency of the open-circuit voltage on temperature and actual aging state of lithium-ion batteries. J. Power Sources, 2017, 347: 1-13. 